\title{
Spectrum Allocation in Cognitive Radio Networks Using Genetic Algorithm
}

\author{
G. Suman Shashank, Noel Mathew, Kishore V Krishnan
}

Vellore, Tamilnadu, India

\begin{abstract}
In this model, we present a method in which the Dynamic Spectrum gets optimized for its usage in Cognitive Radio Netwocts. Firstly, based on the Interference Temperature model, we determine the interference constraints and there is a check on the transusion power of the secondary users. We then formulate the SINR by considering the noise spectral density of the Environmentrand the Transmission Power's of the surrounding neighbors. Using the Shannon formula, we calculate the co-channel interferexee between potential links on each channel. Next, using Genetic Algorithm, we formulate the spectrum assignment problem. Genetic gyorithms are adaptive heuristic search algorithm based on previous and historic results obtained earlier. Although random, GGु\&tic Algorithms exploit historical information to direct the search into a set of better solutions. It is observed that, the total network supacity is improved by a significant margin Bio inspired Algorithms have always been promising in giving Optimum solutions.
\end{abstract}

Keywords: Cognitive Radio Networks, Secondary User, Primary User, Spectrum Allocation, Interference Tegiter

\section{Introduction}

A considerable amount of algorithms have been introduced in the domain of spectrum access in Cognitive Radio networks. These methods have risen by the arrival of CR Technology and change from the usual fixed spectrupe assignment to the newer spectrum access technigas. These models aim at improving the spectrum efficiend by regarding opportunistic and dynamic spectrum for the Secondary Users. Considering Cognitiog and Configurability as two of the main features Ald capabities of the CR node, it comprises mostly two types of 3 hases. The first Phase revolves around by having thectrab environment sensed.

The CR user captures the information of the bands and based on quality level, classify to chethnels available. The second phase comprises ang af spectrum allocation which apart for safying interference constraints, allows availagote chtnnels to 8 ' in such a way that the spectrum enfierey is matizos. Most works, disregard the Qg parmeters of sleh as SINR and capacity and simpldary thaximize theanumer of active links between SLOS us Integer Linear Prograknix (BILP). T, ey sely on

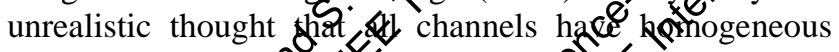
nature with regargho Q that using of number simultaneous communication links around applying a higher rate. On each would lead to a higher total capacity. The hererogeneity of links have been considered in a 3 step manger by computing the maximum allowable power reovived from receiving nodes and the actual transmission o power at transmitting nodes, spectrum between SU's is classified to high and low power transmissions. Basically, the channels have been classified into two categories according to their transmission power and haven't captured the exact link capacity. Models based on Interference range have also been used but are not accurate enough though. These models usually define an interference range in the surroundings of the receiver where no transmission is allowed. The conservative nature of these models might degrade the spectrum thtireg methods. New Link capacity aware algorishm are used tQ2maximize the capacity to such and the that it inclupes different QOS parameters like their SơR and bandwidt along with tentoera oure interferengs madês.

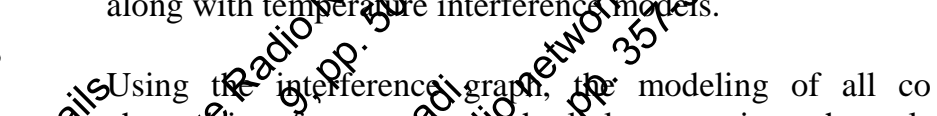
channos $e_{\text {interference }}$ the Inks on various channels tak place. Following allocation is formulated in

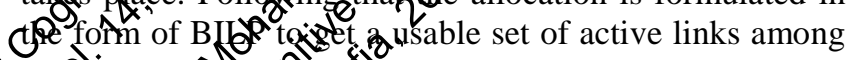
o' other links in tho gesh in such a way that maximum sapacity is attainged To tackle the high complexity of these fields, ove ofe dix Tree structure in which we have scatided_fsarts sof the solution space removed. Following that the propem is formulated onto a radix tree search the hophtering links in the graph.

\section{तथ}

10 O the past two decades, there has been a rapid increase if the use of wireless applications, which in return leads to the need of more Bandwidth. Unfortunately, that situation has lead to a scarcity in the Spectrum, which arises due to the inefficient use of EM spectrum. EM Spectrum is the electromagnetic radio emitted from different sources, which accompany its own frequency and wavelengths. The FCC (Federal Communications Commission) controls the EM Spectrum and only licensed users can use the EM Spectrum. The use of these spectrums varies from locality to locality. The misuse often leads to the efficiency decreasing throughout the spectrum amongst the wireless networks. Hence cognitive radio has been introduced to take care of these spectrum allocation problems. It is a kind of smart radio, which can adapt and change its behavior according to the environment. There can also be a borrowing of unused spectrum from the primary user to a secondary user. Although, this kind of an arrangement must make sure that the primary user's communication is not interrupted. Quite a few issues can arise while the spectrum is handed over from one user to another. The device must be intelligent to sense any holes in the Radio environment. It must be able to understand the requirements of application. Studies have proven that the QOS constraints aid in giving efficient wireless communication. The proper sharing under the basis of 


\section{International Journal of Science and Research (IJSR) \\ ISSN (Online): 2319-7064}

Index Copernicus Value (2013): 6.14 | Impact Factor (2015): 6.391

agreement of the unlicensed spectrum with the licensing authorities should be met with, to use spectrum without harming the licensed users. It should also be self aware of it's own operational capabilities.

\subsection{Objective}

In this model, we present a method in which the Dynamic Spectrum gets optimized for its usage in Cognitive Radio Networks. Firstly, based on the Interference Temperature model, we determine the interference constraints and there is a check on the transmission power of the secondary users. We then formulate the SINR by considering the noise spectral density of the Environment and the Transmission Power's of the surrounding neighbors. Using the Shannon formula, we calculate the co-channel interference between potential links on each channel. Next, using Genetic Algorithm, we formulate the spectrum assignment problem. Genetic Algorithms are adaptive heuristic search algorithm based on previous and historic results obtained earlier. Although random, Genetic Algorithms exploit historical information to direct the search into a set of better solutions. It is observed that, the total network capacity is improved by a significant margin Bio inspired Algorithms have always been promising in giving Optimum solutions.

\subsection{Motivation} 1.2 Motivation
Primitive spectrum access was confined only
users where only the licensed users were autsorimaryed to the spectrum. In this spectrum access fechniqu the spectrum wasn't utilized completely, hence resited in white spaces and spectrum holes. In order to thtlize the spectrum to the fullest, we explored into the domain of dynamic spectrum access techniques. The congept secondary user comes in in the former techniget, yofere the secondary users to communicate with eash ober use the white spaces. All these circumstates emotivated research scholars and scientists to opringation order to maximize the spectrum acgrs. Thus, unlifshs users can access the spectrum "completely, thensthe primary users are not using its hisoshould p.dore in way not to counter the functigning of the primated by imposing interference ginthent The dynand access is implemente 5 by insorporating coesnitio radio in

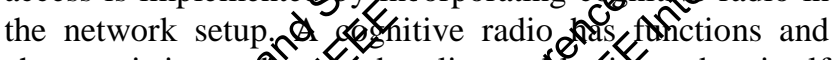
characteristics as ब9ि scussed earlier enflest to adapt itself to the drastic ghanges in the sperup Environment. The spectral effency of Wireless hetrorks is improved by dynamic to utidese appropriately in order to meet the Quality of Senice requirements of different applications. A cognitive seldio is an independent and self-sustaining system, which $1^{O}$ eases the work of spectrum allocation process.

\subsection{Background}

\subsubsection{Architecture:}

A Cognitive Radio Networks comprises of primary and secondary users. Each primary user is associated with at least one primary base station, which together forms a primary network. Primary users are the authorized personnel to utilize the spectrum, hence are called licensed users. Base station is the only source by which, primary users exchange information amongst each other. On another note, Each secondary user need not be associated with a base station, but all of these secondary users as a whole form the secondary network. Each secondary user can access the spectrum through its secondary base station but is limited to the range of their respective base station. Since the secondary users shouldn't intervene with tha functioning of the primary users, they are incorporzte with Cognition abilities. In other words, Secondar tisers should inhibit the usage of the Spectrum as sern as it senses a primary user within its proximity.

\subsubsection{Characteristics:}

1. Radio Environment Sensting: Cognitive Radio Networks adapts itself to the spectral environments. It informs the gor ner nembers of the network about its changed mode $\mathcal{F}^{2}$ opation. Hence, it is best suitable for environmeots that drastically change with time.

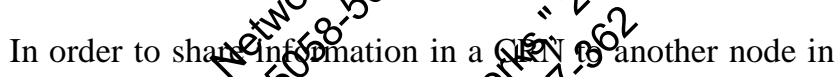

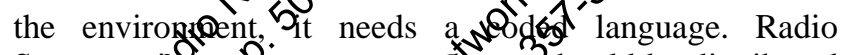
Spectrum 20 a serce resource encen should be distributed evenlyein a Qtniform अंde The Qefore, many algorithms haveerievelop to the spectrum and allocate

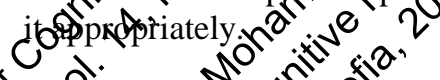

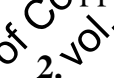<smiles>[C]1[C+]=CC=C1</smiles>
Fpactions: 5 के practions:

Spegxum dolestre highly required to be sensed in order to profentantestrence. The technique incorporated by the orimary is the most efficient in terms of spectrum Shese techniques are divided into interference obas detection, transmitter detection and co-operative detection.

To ensure the user's communication requirements, it is highly necessary to capture the best available spectrum. A collective decision must be met with to determine the best band spectrum that would meet or fulfill the QoS in the range of all bands. This intricate procedure is known as Spectrum detection and Spectrum analysis and fall under the domain of Spectrum Sensing.

To avail the best frequency bands for operations and transferring of information, it is required to exchange the operating frequency. The shift or transfer onto another frequency must be smooth. This shows good prospects of Spectrum Mobility.

A major Area of concern in today's spectrum world is the inability to share the spectrum properly. These issues directly correspond to MAC problems. Hence the idea of Spectrum sharing needs to be taken as a serious issue, as its proper application can ensure better usage of the Spectrum. 


\section{International Journal of Science and Research (IJSR) \\ ISSN (Online): 2319-7064}

Index Copernicus Value (2013): 6.14 | Impact Factor (2015): 6.391

\section{Project Description}

\subsection{Basic Concepts in Cognitive Radio:}

The QoS is defined as the required set of qualitative and quantitative characteristics of the communication system required getting the proper or desired functionality of application. A QOS parameter can be based on human perception of different media and its attributes. Such as time dependence and symmetry.

Long back the software defined Radio was introduced and is defined as a terminal that is competent to operate with a lot of bandwidths. They are able to support different standards such as GSM, OFDMA, CDMA, and WiMAX. They promise the best solution for best connectivity problem, but the requirements of QOS by an application is still under consideration. Thus cognitive radio is introduced.

$\mathrm{CR}$ is a radio that can read and understand context in which it can tailor the communication process along with understanding.

If a SDR needs to be a CR then:

1. Management and optimization of spectrum

2. Optimization and management due to consisting variety of wireless networks.

3. Aiding humans using EM Resources.

There has been a rapid increase in the applications, which in return leads to the need ofmore Bandwidth. Unfortunately that situation has istd to a scarcity in the Spectrum, which arises due to thefficied use of EM spectrum. EM Spectrum is the electromaticts radio emitted from different sources, which accomalny own frequency and wavelengths. The FCE $e^{e}$ (Ederal Communications Commission) controls the fog spectrum and only licensed users can use the EM Spitetruef.

\subsection{Behavior and Cognition Capąkingtiga}

For efficient communication 0 ती. today's world follow a differents ature in theicselyorior. For e.g.the power imbjncedcan be nulliford deetween different users by thesproper use of the geitpuxepower. A phone can adapt signal regardles ${ }^{2}$ W-max networkes maintain good throughput. It 9 Pso has a decent lity factor and the characterist res of its signals have the \&ility to adapt.

\subsection{1 \$ehavior:}

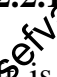

much increased and can adapt to many types of parameters

such as the beam pattern of an antenna, the power, the modulation technique, coding technique and the operating frequency. It also takes into consideration the battery and processor usage. The adaptation can occur in planned and unplanned manners. The cognitive radio device recognizes the radio environment to bring out further more potential in communication.

\subsubsection{Cognition capabilities:}

The different abilities as seen before allow to define the must have radio capabilities. These are regarded as the cognition capabilities. The various capabilities include reasoning, learning, sensing and awareness. Due to these parameters the Cognitive radio device can be defined as a radio that is capable to comprehend the different environments that it gets involved in, its requirements, its potential and its regulatory policies.

In Lehman words we can say that it has the cote ability to be self-aware and completely know its environment and its variant nature over a perigof time. It executes the received information and nofes its own decision on how it needs to make neces\&ary arrangements to put together a successful communicgon process.

\subsection{The Cognition Cycle:}

Cognitive Radio is the rado thas in itself the ability to reason with regulaxpry Splicies, different user requirements, be of its potentials and requirements. It the \& \& for a CR, therevistisba need to make pos per decision on which way action must be mase 35 remembering this factor wa ean pinclude that gognitive radio has the ability observe, deciaté a a act.p م.

talk art.gs arvertion, it involves the process of

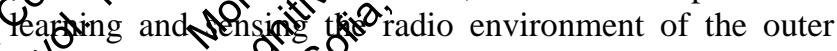
world. The Observations recorded are given as the input into the of on the of of the mechanism, which is inculcated in it throu ghaevesoll algorithms.

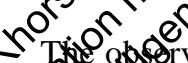

arvations and decisions decide the complexity of a OProsess in Cognitive Radio. And those parameters can decide if or not the process shall occur as complex or noncomplex procedure using the involvement of past knowledge or future knowledge and few probabilities. Hence it is very important to properly design so that intelligent and appropriate decisions can be tallied.

According to the cycle, it starts at the observation end and then goes further traditionally. The cycle,although, actually starts at the "act" end and that is due to logical reasoning. It is so to know what actions are of importance and has the possibility to occur. And this needs to occur before we get to understand what decisions need to be taken and what decision needs to be made.

Hence the important three steps include as;

1. Taking an Action

2. Making an Observation

3. Making a Decision

\subsubsection{Taking an action}

These are the actions that can bring out a certain set of performance out of a cognitive radio or the different alterations and actions of all forms. The action process depends on the observation part and the necessary 


\section{International Journal of Science and Research (IJSR) \\ ISSN (Online): 2319-7064}

Index Copernicus Value (2013): 6.14 | Impact Factor (2015): 6.391

indications for alterations are provided by it. The CR can be made efficient to achieve a desirable goal by altering various parameters such as the pattern of antenna beam, the power, the modulation techniques used, the bandwidth, frequency etc. The parameters must be decided first before proceeding and the $\mathrm{CR}$ must be aware of all the actions possible before it goes onto perform a certain task.

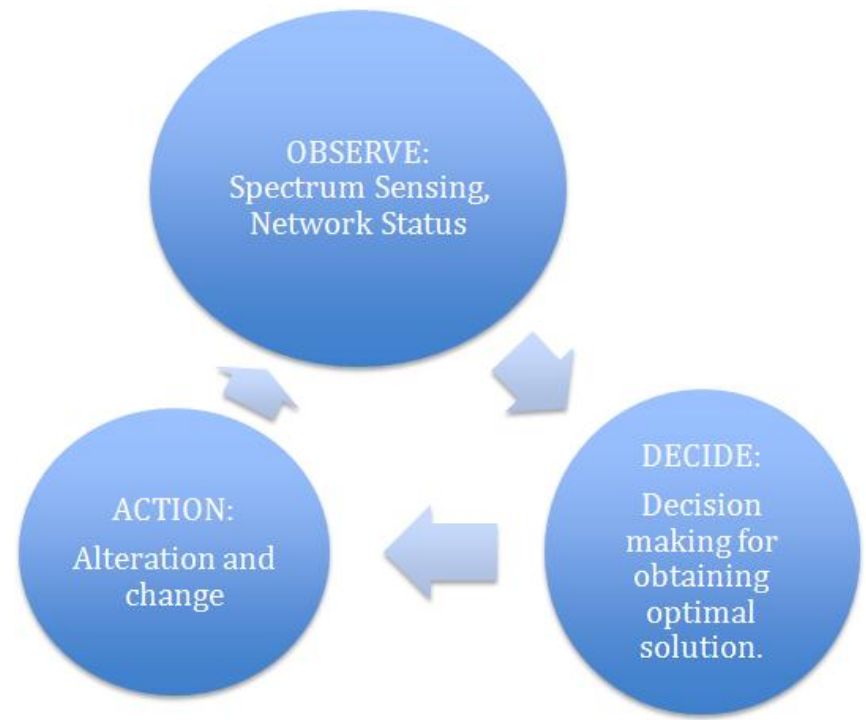

Figure 2.1: Cognitive Cycle

\subsubsection{Making and Observation}

To get a certain level of performance, the radio are altered in many ways. Clues and few hig are attached along with these parameters to enje the necessary alterations. The observations thag \&le talligg throughout the process help in aiding the radio with the necessary clues. This area is used as the input and holp determining which state the radio is working ofn ofter deciding the state the decision is made and ofte astion is taken accordingly.

The observation part involves paind derstanding $e^{e}$ the requirements of the users and the policiess involvad Ags it or

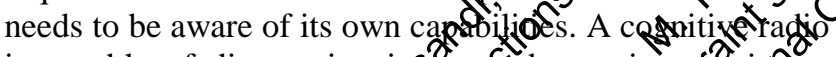
is capable of discovering itsound observationg if it can receive tallied observation ing external souros (5.

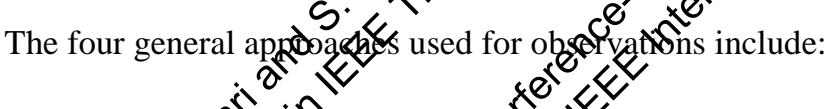

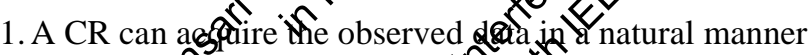

2. With thepfikes of GPS DelfesGused, the hardware device tian be used to make certain observations. These are tew of the unnatural forms of attaining certain Pervations of the environment

S By using the technique of special signal analyzing the observed data can be acquired

4. By considering the environment it is operating in and learning its parameters.

The last seen method is the most analytical of all methods when it comes to making remarks.

As a part of making observation, necessary spectrum sensing needs to be done to come up with certain conclusions regarding the environment the radio is operation in. Generally the spectrum sensing part is synonymous with the cognitive cycle.

\subsubsection{Spectrum Sensing}

This technique mainly involves in ruling or detecting the signal transmitted and of interest of the CR Receiver. With this method, the CR is enabled to spot or find the existence of other radios or find whitespaces in its concerned radig 0 Further into Spectrum sensing, it is divided into furto aspects such as Accurate Sensing, sensing the Approtiate Range and In time Sensing.

\subsubsection{Accurate Sensing}

In order to detect the spectrum accuratofy, the CR must be made smart enough. It can have a nissed detection and a false alarm. For E.g. It would dee a primary user in the environment when there is nop primary user in reality. The other scenario is pretty werche opposite, as the CR would not detect a userphen $\mathrm{P}$ in reality if really present. Both of these scenaris to inefficied communication and lead to problens sus as interference

\subsubsection{Sensing in the appropriaterage}

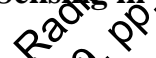

It helpe in ipcreasing oे $^{2}$ onproping the sensing. Every

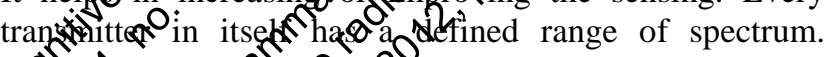
Hothethis deford. Gegore signe? The depends on the receiver's sennsitivity pand doe tronsmitter's power. Both primary and secondar so àble to sense within a certain range as theyjuavereckilers having such level of sensitivity. If sering os degte with the proper range in hand it can avoid onakn such as the false alarm scenario or the $F$ nosisedetection.

\section{oc $x^{e}$}

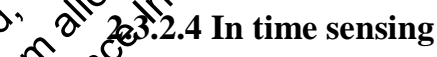

It majorly senses the primary user in the spectrum. It can be classified into two terms, the first being white spaces and the second being return of the primary user. White spaces, The first term, stresses on the free time of the primary users which ends up in creating spaces or holes in the spectrum. Therefore it is required to find the opportunity to communicate and finding these holes help in enabling that. The opportunity to communicate is lost if a lot of time is taken to sense these holes. The second term applied, the return of the primary users mainly addresses the return of the licensed user to the spectrum. It is highly required to avoid the unwanted interference between the secondary and primary users. There is a high chance of interference to occur is it takes too long to observe a primary user and that wouldn't be suitable for efficient and enhanced communication process.

\subsection{Selecting a Proper Interference Model:}

The primary step in building a CRN is to choose an appropriate interference model. The foremost thing in constructing an interference model is to build a channel propagation model relative to the radio environment. We 


\section{International Journal of Science and Research (IJSR) \\ ISSN (Online): 2319-7064}

Index Copernicus Value (2013): 6.14 | Impact Factor (2015): 6.391

shall consider deterministic path loss, large scale and small scale fading which are the propagation effects in channel models. Under the assumption that there aren't any obstacles in the radio environment; we ignore the fading effects and consider only deterministic path loss effect in our channel model. The next stage is to establish an appropriate transmission channel model, which exhibits the effect of interference on the received signal at the receiver end. Primitive channel model were derived from collision channel model where if two or more transmitters try to send a signal to a receiver at the same time. All of the signals would be summed to zero due to the collision effect.

In the recent new era of models, capture channel model is proposed where if one of the received signals is strong enough compared to the other signals that signal would win over the other and reach the receiver. Vulnerability circle capture model and power capture model belong to the above category. In the vulnerability circle capture model for an $i^{\text {th }}$ transmitter signal to be successfully received, its receiver power must be more than the power of any other received signal by a factor $\beta$

\section{$\operatorname{Pr}, i / \operatorname{Pr}, j>$} for $\rightarrow j=1,2 \ldots . . . n ; j \neq i$

Let us consider a model where there is unifo power level for all the transmitters. according to the definition of vulnerability circlentiodel, the radius of the circle is $r=\beta^{1 / n}$

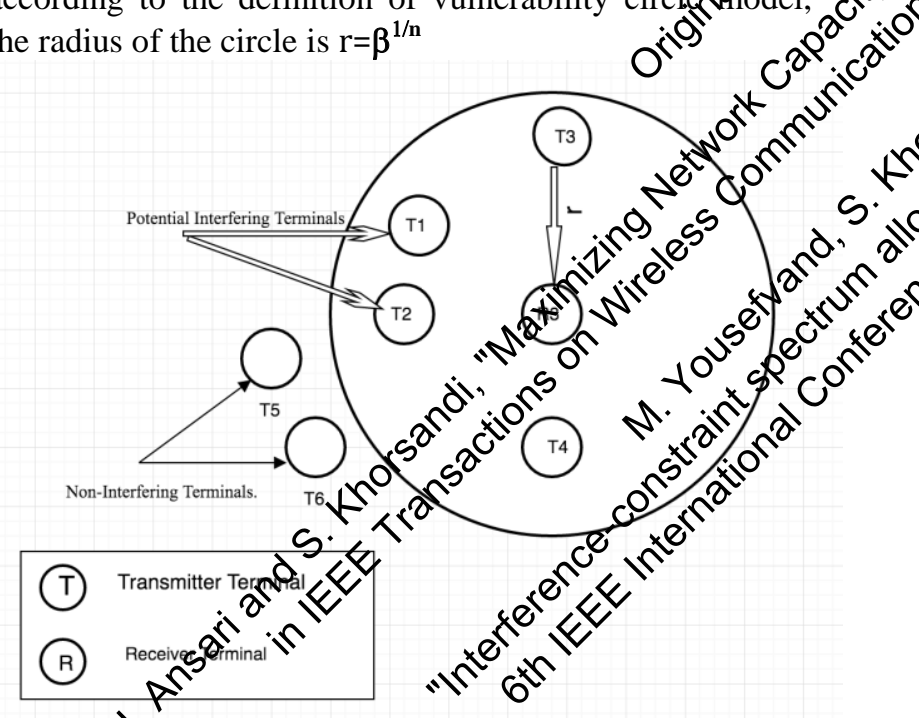

Figure 2.2: Vulnerability Circle

Ine the above diagram, the vulnerability circle diagram is elemonstrated. The transmitted signal from $T_{1}$ is intended

to be received by $R_{1}$ but it is interfered by the signals transmitted by other transmitters within the vulnerability circle.

This is a primitive model, which imposes huge restrictions on the transmitted terminals located around the receiver and results in degradation of spectrum efficiency.
For example, if we take a transmitter node $\mathrm{T}_{3}$, it's signal is intended to the receiver $R_{3}$, without causing any interference on any other receiver. In present times, according to practical power capture models, a signal is set to be received successfully at the receiver end if the received power supersedes the power of the accumulated signals from all other receivers by a factor $\square \mathbf{p}$.

Mathematically we can deduce signal to interference ratio as :

$$
\operatorname{SIR}=\left(P_{r, m} /\right.
$$

$n$

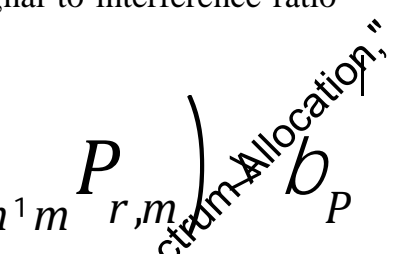

In the above equation, the received poye at the receiver end of the transmitted signal $i$ is $\mathrm{P}_{\mathrm{r}, \mathrm{i}}$. $\mathrm{n}$ a more realistic scenario, we need to consider additye noise power which is denoted as $\sigma^{2}$, mathematicye signal to noise interference ratio

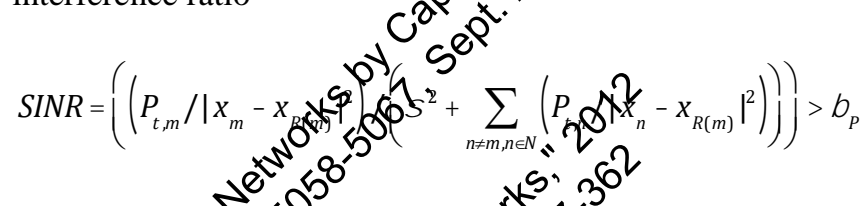

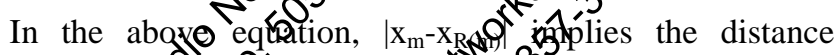
between transnoper $m$ and itsereceiper. The bandwidth in the abge modrel is at of clotant

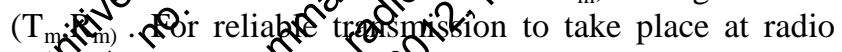
egorranent, the ofingerony given link should exceed a

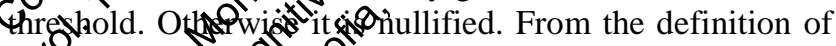
Snannon'sformof for link capacity, the data rate for the

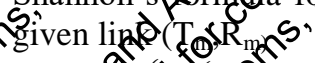

$W_{\text {की }}$ औौ

के 0 pregents channel bandwidth and $\mathrm{N}_{0}$ denotes the noise ofectity density.

\section{Technical Specification}

\subsection{Genetic Algorithm:}

Evolutionary algorithm was found to be promising in solving the optimization problem. Hence we have opted for genetic algorithm to maximize the network capacity, thus optimizing the spectrum as a whole.

\subsubsection{Approach:}

The algorithm is carried out by random choice of chromosomes that have a particular trait which are computed through generations. The fitness of a given chromosome at a particular generation is evaluated on the basis of a random probabilistic calculation. Calculation of fitness of chromosomes is based on individuality, the process continues for several generations iteratively till we reach an optimum solution.

\subsection{Stages of Genetic Algorithm}

\subsubsection{Initialization:}

$\mathrm{N}$ chromosomes re generated randomly which form the initial population. Probable solutions for the given problem are part of the population stated above. 


\section{International Journal of Science and Research (IJSR) \\ ISSN (Online): 2319-7064}

Index Copernicus Value (2013): 6.14 | Impact Factor (2015): 6.391

\subsubsection{Fitness Measure:}

This stage includes computing the fitness of chromosomes, which constitute the initial population.

\subsubsection{Construction of New population:}

Selection: A set of chromosomes is selected based on the criteria of fitness from the available population

\subsubsection{Crossover:}

New progeny are part of the upcoming generation by the method of crossover. Progeny are formed or reproduced based on the probability of crossover

\subsubsection{Mutation:}

The chromosome structure of the offspring is converted from string to binary format and the binary values are toggled at a particular point to retain the diversity of the upcoming generations.

\subsubsection{Stopping Criteria:}

This iterative method is run through multiple times till we reach an optimum solution. It is also found that, the stopping criteria are relative to the complexity of the chromosome structure.

\subsubsection{Roulette wheel selection:}

In this selection process probability of selection of a give chromosome is directly dependent to its fitness. Copster $\mathrm{M}(\mathrm{I})$ as the fitness measure of a chromosome, ben the selection probability of this chromosome is gijot as $\mathrm{P}$ (Ne which can be mathematically formulatgros fitno measure $\mathrm{M}(\mathrm{I})$ of the given chromosome divid by summation of all the fitness measures of ' $\mathrm{N}$ ' nimblor of chromosomes present in the solution domaim roulet wheel selection process, each chromosome is estipated, with the sum of fitness of all the chromosomes ad the chromosome with greater probability has morg etran of being selected. Using any of the aboy-mentioned procedures reproduces the child chromosomis.

Table 3.1: Roulette Whegrelection

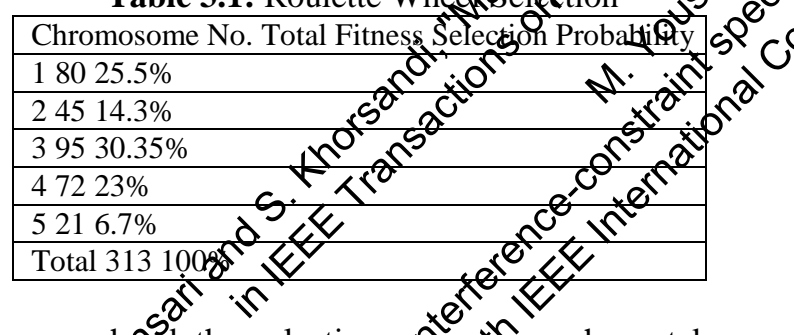

To comprehend the selection we have taken a possible set of solutions of 5 chromosomes with their fitness reasures and evaluated probabilities of selection as givg in the above table. It is evident from the table above theat the $3^{\text {rd }}$ chromosome has maximum fitness measure otmong the whole set and the $5^{\text {th }}$ chromosome has the least fitness measure and hence the corresponding selection probabilities.

This selection process has been named Roulette Wheel because, the wheel is whirled $\mathrm{N}$ times for $\mathrm{N}$ number of chromosomes in the solution domain.
Since the first chromosome has the maximum selection probability, it shall occupy maximum portion on the wheel whereas the fourth chromosome with least selection probability occupies the least portion on the wheel. While the wheel is spun, the probability of selecting the first chromosome, which has a larger area, is always more than the rest of its counterparts. Thus this whole process eliminates the selection of the poorest chromosome almost unlikely.

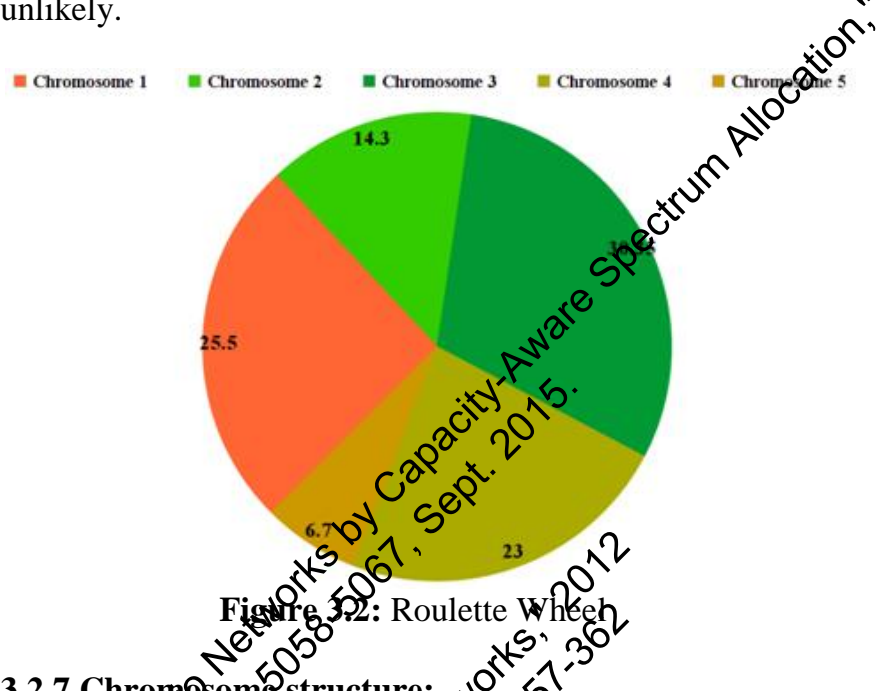

The proges in upon secting an apporis chosome structure. Each chratosone compeses diferent genes. Each gene in a cbonalsome depictge some characteristics of the RF

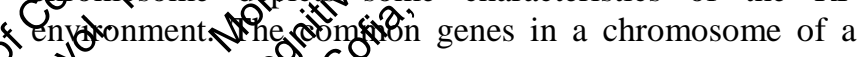
RF envirompmentale sognal power, data rate, and operating ofrequency. Ghromsomes are the basic elements of Gengic mor be showcased in a way the the probable solution is arcempanted with it.

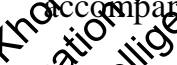

19 9 histost generalized form of representation is in the form d binary string. The genes of a chromosome are built e using basic radio parameters. The most essential condition is the variety of chromosome's necessary process. Power is the greatest necessary radio parameter for communication. The quantity of power is completely relative to specific applications. Extreme powers which maybe too high or too low maybe undesirable for effective communication. Thus it is mandatory for power to be in optimum range, which can boost the chances of reliable communication. Signal power is generally kept low to remove flaws in the communication.

\subsubsection{Binary Encoding of Chromosome:}

In them mutation state of genetic algorithm, chromosomes are binary encoded. Each gene in a chromosome is transformed from decimal to binary for the mutation to carry out. Soon after them mutation process the genes are changed back to their original decimal forms.

\subsubsection{Fitness measure:}

An objective function is defined to determine the standard of each chromosome that comprises a part of the Genetic process. The fitness measure is directly dependent on the nature of the problem. A new generation in the iterative cycle is formed by the application of the fitness measure. 


\section{International Journal of Science and Research (IJSR) \\ ISSN (Online): 2319-7064}

Index Copernicus Value (2013): 6.14 | Impact Factor (2015): 6.391

In fact, it replicates a filter which restricts individuals that are not meeting a particular level of fitness So that the chromosomes that are deviating from the optimized solution will be truncated. This stage is followed by selection crossover and mutation.

\subsubsection{Crossover:}

After the selection stage in genetic algorithm, fit chromosomes are chosen and the next stage is to takeout crossover. Crossover is a technique by which dealings of any two chromosomes can be interchanged with each other to form two new offspring. There are multiple crossover techniques, namely single point, multi point, two point and uniform crossover. Research shows that, two-point crossover technique yields better solutions relative to other techniques. Generally, crossover rate is between $85-95 \%$.

Random cluster of crossover points are chosen in the chromosomes, which will segregate them into parts having different parameters. Crossover process is followed by mutation. The random value that is generated is compared with the mutation probability if it is found to be lesser, than a reversal of one of the bits in the process. It is ideal to keep the process rate low so that it doesn't interfere with the fitness level and nature of offspring.

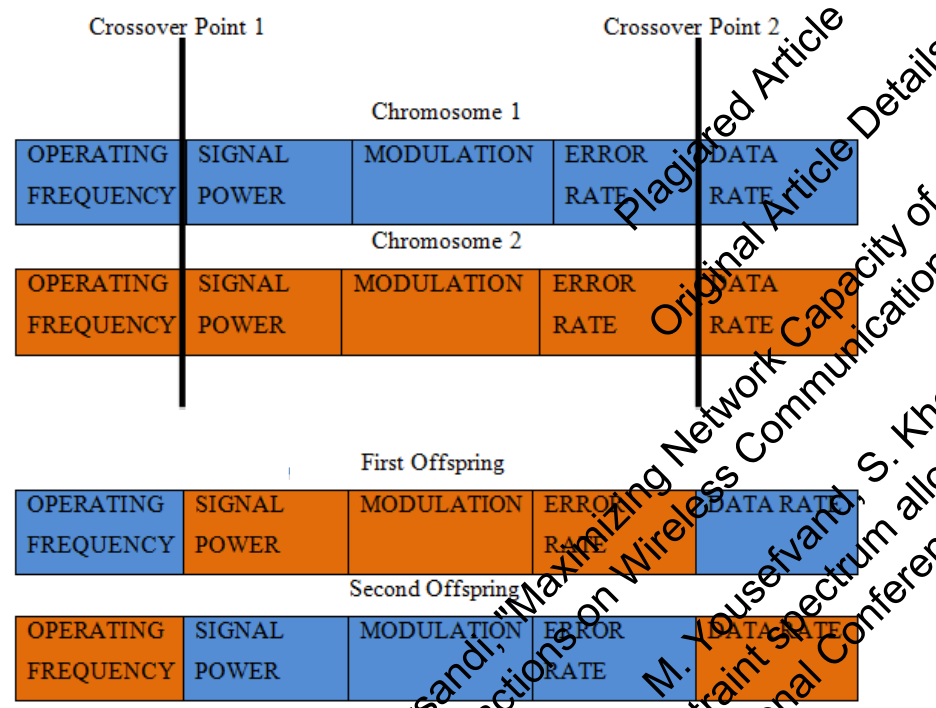

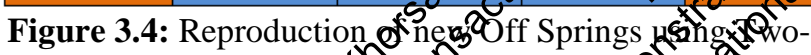

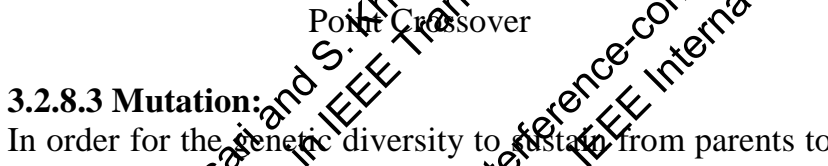
child chromosemes, mutation is essential stage in GA. Mutation is carried out on the genes of the offspring which are just produced after the crossover, by toggling binakf bit of $0-1$ or vice versa. Unlike crossover, mutation cant be formed with decimal representation. Hence, Shromosomes have to be converted into binary form. There may be adverse effects on the chromosome structure of the offspring hence the mutation rate is kept low which is around 2-4\%

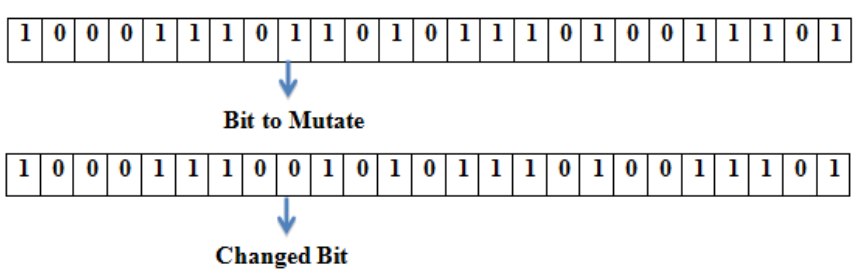

Figure 3.3: Mutation Process

3.2.9 Stopping criteria
This criterion is completely related to the optimization problem. When a solution with desired values is rescied then the stopping criteria comes to play. It is a gommon observation optimum results are produced in boveen 6090 generations and the genetic process pacgodown with increase in the number of generations. Fig 8 ss measure is imposed on the final generated cluster the most desirable one is chosen as optimum. Genetic atorithm is associated with random values, so there is afairshance of choosing wrong results. GA is known tgoprace a set of optimum solutions rather than a singlegolytion.

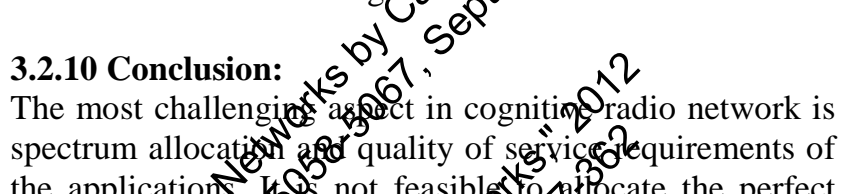

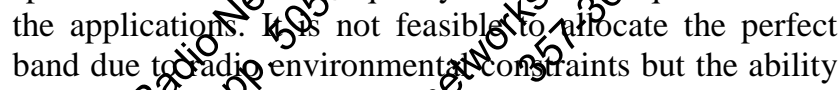
Sto assignRtheogest band onof thexisting bands is a topic to beseasched. Cgesizat alowt all these constraints, we hayelpeposed a ectgin apocation solution for cognitive

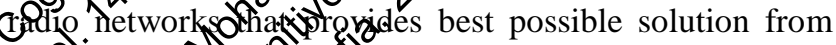
o' tho Oavailable set of eattions. Without compromising the कOS regsiremats ${ }_{5}$ Genetic algorithm doesn't give $100 \%$ efficiens squgedue to its random behavior but choosing a sedededyutetion can rectify this. $s^{2} \sqrt{2} \times$ (a) segulation, the first portion of population is and generated. And the second part is generated using the information of first. This reduces the randomness \& population. The seeding of population can cause another issue that is premature convergence. Premature convergence can be countered in the following ways. By increasing the rate of crossover and mutation, we can decrease the probability of pre convergence. But this will increase the iteration time and also randomize the solution.

By increasing the initial population, we can decrease the probability of premature convergence. However, execution time will increase corresponding. Although the above solutions are proposed, there is uncertainty in radio communication. It is concluded that the proposed solution is favorable for removing white spaces and best possible usage of the spectrum in cognitive radio networks.

\subsection{Spectrum Allocation:}

The heart of our project work revolves around Spectrum optimization in CRN. Cognitive radio receiver senses a radio frequency environment and a spectrum hole is allocated to a requesting user without compromising with the quality of the service requirements

Spectrum allocation test: is performed for the given spectrum and if the resulting parameters meet the users QOS requirements, the process is stopped; if not, multiple 


\section{International Journal of Science and Research (IJSR) \\ ISSN (Online): 2319-7064}

Index Copernicus Value (2013): 6.14 | Impact Factor (2015): 6.391

tests are carried out until we reach an optimum solution. Spectrum allocation regulations are to be followed which the regulatory authorities authorize. These norms serve to decrease the interference in the spectrum bands and also to meet the QoS Requirements.

Spectrum allocation test: is performed for the given spectrum and if the resulting parameters meet the users QOS requirements, the process is stopped; if not, multiple tests are carried out until we reach an optimum solution.

\subsubsection{Making a decision:}

The decision making process is the most pivotal aspect in determining the performance of a cognitive radio. The distribution and the usage of radio resources are the elements that require the most precise decision-making. It also helps in enabling fair communication amongst other users.

\subsubsection{Optimization:}

The best choice of the existing options, which is most feasible choice, that enhances usage and also enables to reach the desired goal with high efficiency.

Optimization hovers around three different types of scenarios, which include;

1. Optimization Goal

2. Available options

3. Determining the best option

3.3.2.1 Optimization Goal:
Selection of efficient systems in such a way that as the effective communication is maximized.

\subsubsection{Available options:}

They comprise of a set of feasible solutions or gtiono of which the best option is chosen in ord to seach a particular goal.

\subsubsection{Determining the Best Optipo}

A fitness test is set to determine.the be (1)

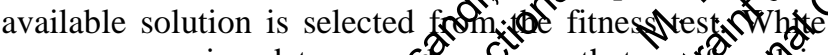
spaces are assigned to secoperarydtsers that argstoun in spectrum sensing. Anothor aspect of this optization is putting a check on tbs.poner consumptien. elfeedback mechanism is requijed fevery user ipa gits cognitive radio to control ke par by itself. ges seguirements of a given commughe ation system is the above power control mectrinism.

$$
\overrightarrow{0}
$$

\subsubsection{Merits of Genetic Algorithm}

The 9 key utility of Genetic Algorithm is its computation ffility, which can reduce the simulation time. It can 10 optimize for many number of variables. It doesn't give a single solution but a list of optimum solutions. Fast coincidence is another utility of Genetic Algorithm that can meet the solution in minimum time.

\subsubsection{Demerits of Genetic Algorithm}

A complexity is involved during the encoding and fitness measure stages of GA.
Given, the number of variables is high; It would lead to cumbersome process in applying the algorithm. Thus the simulation time gets longer. In situations like these other techniques prevail over Genetic Algorithm. In short, the algorithm faces major constraints with respect to number of variables. Although, this particular drawback can be resolved, Given, Sophisticated hardware architecture has been applied.

\section{Design Approach and Details}

4.1 Methodologies and Design of Spectrum Allocapton: Our proposed model consists of five stages. these stages form the cognition cycle. It is named cognition cycle because of the ability of cognitivespldio nodes to sense the spectral environment and denamically adapt itself to the new state of the environmsit. In the spectrumsensing phase, existing frequengy ghannels are being monitored by the SU's to get infortin such as the noise and interference level of the sicât layer

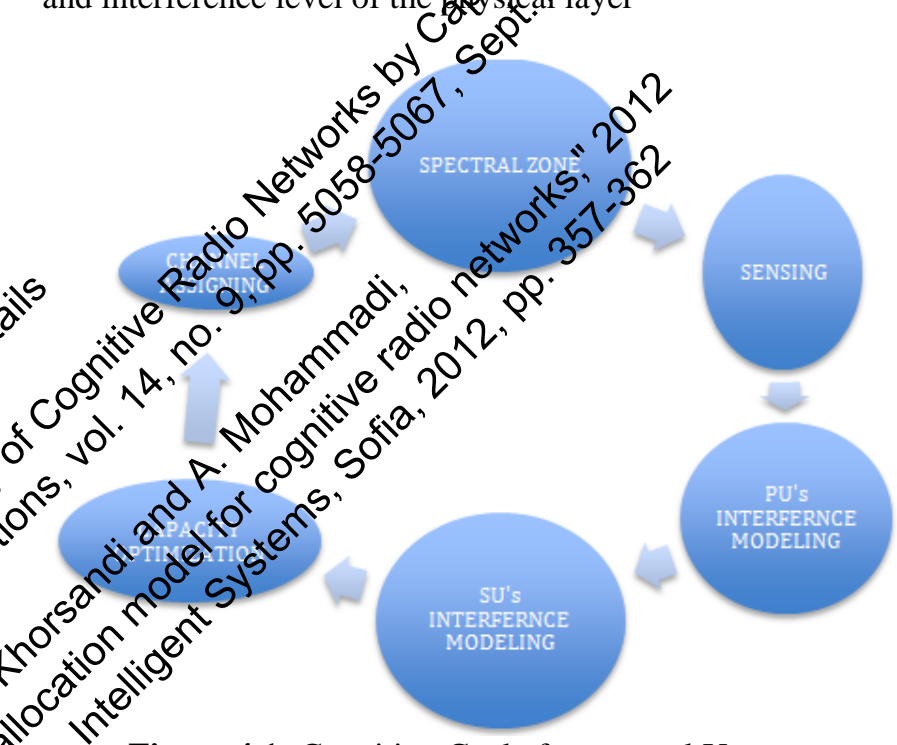

Figure 4.1: Cognition Cycle for spectral Users

Secondary users are authorized to increase their transmission power in such a way so as to not interfere of the primary users by being aware of their position and interference temperature thresholds, so that the constraints of the primary users should be met.

In the second stage, On each channel, the transmission power of the secondary users are increased; given that interference constraints on all the affected primary users are met. By determining the transmission power and positions of users at their respective channels, we can calculate their capacities by making use of Shannon's formula. Since there exists Co- Channel interference between adjacent channels, hence we need to capture this first before applying the optimization. It is not possible for all the potential links to be active simultaneously in a given channel; we determine a feasible bunch of Active links operating at the same time in order to maximize the network capacity. In the final stage of channel assignment, after identifying a bunch of non-interfering links, the remaining channels will be allocated to the secondary users correspondingly. There are two sub stages in interference control of cognition cycle. 


\section{International Journal of Science and Research (IJSR) \\ ISSN (Online): 2319-7064}

Index Copernicus Value (2013): 6.14 | Impact Factor (2015): 6.391

In the first sub stage, being cognizant about the primary users events we finalize maximum permitted transmission power for secondary users to assure that the primary users that their interference constraints. In the second sub stage, we make sure that interfering links on each channel does not get activated simultaneously, by modeling the co channel interferences. Secondary users can raise their transmission power between the lower and the upper bounds; the upper bound is the maximum allowable transmission power. But SINR should meet its threshold level for reliable transmission.

\subsection{System design and Analysis:}

Let us assume that there are $\mathrm{K}$ primary users in the spectral environment transmitting to each other through $\mathrm{C}$ channels and $\mathrm{L}$ secondary users. Competing against each other to access the spectrum opportunistically.

Let us define a matrix to determine the availability of link between two users. Mathematically

$$
B_{c}=\left\{b_{m, n, c} \mid b_{m, n, c} \quad\{0,1\}\right\}
$$

$$
\begin{aligned}
& P_{m, c} P_{\max _{m, c}} r: T_{r, c} T_{c} 1 \quad r \quad M \\
& P_{m, c}=\left(p_{\max _{m, c}}+\right) r: T_{r, c}>T_{c}>0
\end{aligned}
$$

Apart from the maximum limit imposed on the power : transmitted by the secondary user, there is also a minimumor transmission power level required for each secondar $y$ user's transmission so that the condition of miphum SINR is met. Each secondary user has to chaose its transmission power from its minimum andsinaximum bounds. Mathematically, we can deduce the gelation as $\mathrm{P}$ $\left[\mathrm{P}_{\min \mathrm{m}, \mathrm{c}}, \mathrm{P}_{\max , \mathrm{m}, \mathrm{c}}\right]$. Having deduced the tran $\mathrm{nmission}$ power of each secondary user $m$ on their ohannels, we shall determine link capacity of each ppential links on these channels. We shall construct matrix $\mathrm{W}_{c}$ as $\mathrm{w}_{\mathrm{m}, \mathrm{n}, \mathrm{c} .}$. Each element in this motix pepresents weight of the link in between transmitterch and receiver $n$ on channel $c$. mathematically we can ooternime it as:

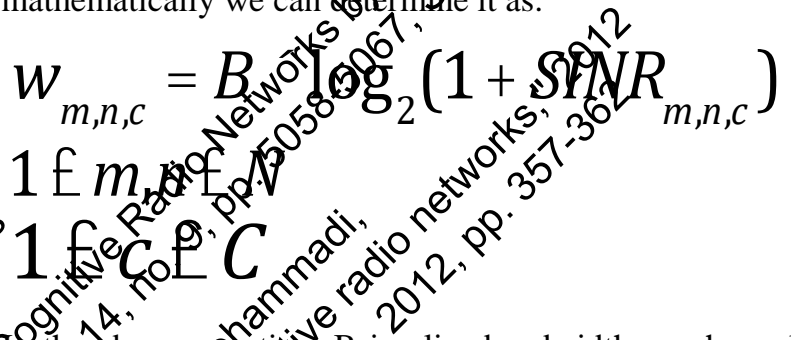

an the above plation, poimplies bandwidth on channel c

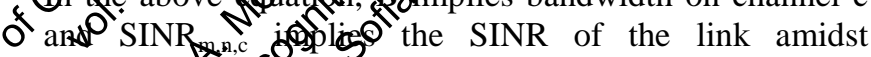
Gransmittor mafted regaeiver $n$ on channel c. we can calculate

SINR the potial links as:

In the above equation $b_{m, n, c}=1$ if both secondary users and $\mathrm{n}$ sense the channel $\mathrm{c}$ as a free channel. If not, $\mathrm{b}_{\mathrm{m}} \mathrm{y}$. In order to infer if the channel $c$ is free or no each secondary user relates its observed interfereng on thate channel with the interference temperature to shold. fact, mathematically, secondary user m senßes the channel free if the following condition is satisfied.

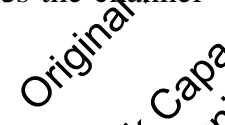

\section{$M$

$$
P_{r_{m, n, c}}+N_{c} T_{c}
$$ \\ $k=1, k \quad i^{r_{m, n, c}}$}

In the above equation, $P_{r, m, n, c}$ is secondary user $\mathrm{m}$ from a signal $\mathrm{b}$ channel c. The noise on the channedis givendy after discovering a set of of eng pil links for sech id the

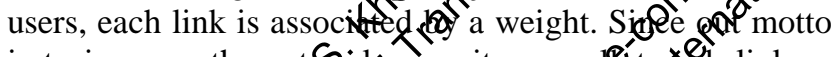
is to increase the network capacity, we difot link as link capacity. Fensecondary users raises its transmission dower for a gid simultaneous $\$$ being in accordace constraints, of the primary users. In Pact, mathematically, we car strike a relation for the transmission power of the secorfary user by determining the maximum allowable tromission power. If the transmission power from the Secondary user exceeds the limit by a factor $\lambda \square \square$ thenthe condition on interference threshold is violated $\square$ Hence we can mathematically deduce the following equation.

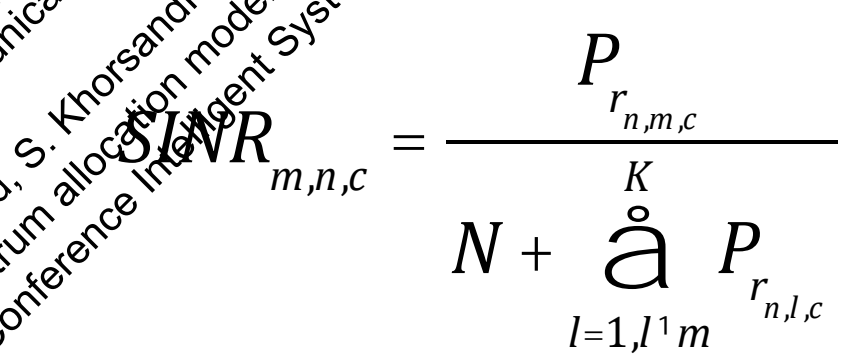

In The above equation, the power received by receiver $n$ is $\mathrm{P}_{\mathrm{r}, \mathrm{n}, \mathrm{m}, \mathrm{c}}$ which is from transmitter $\mathrm{m}$ on channel $\mathrm{c}$. The background noise and the channel are denoted by $n$. The total number of active transmitters on channel $\mathrm{c}$ is $\mathrm{k}$. in order to control the co channel interference on each of the potential links; we construct a matrix to remove all possible conflicts.

$$
G_{c}=G_{C_{m, n, p, q}}
$$$$
\{0,1\}
$$

In the above equation, $G_{c(m, n, p, q)}$ will be taken as unity under the condition that a potential link between transmitter $\mathrm{m}$ and receiver $\mathrm{n}$ intervenes with the link between transmitter $\mathrm{p}$ and receiver $\mathrm{q}$ on channel $\mathrm{c}$. if not, $\mathrm{G}_{\mathrm{c}(\mathrm{m}, \mathrm{n}, \mathrm{p}, \mathrm{q}}$ will be nullified. One link intervenes with the other, under the condition that coinciding transmissions on 


\section{International Journal of Science and Research (IJSR) \\ ISSN (Online): 2319-7064}

Index Copernicus Value (2013): 6.14 | Impact Factor (2015): 6.391

these two links result in the SINR of one of them to dip below a certain threshold SINR required having successful transmission. To avoid non- active links to be part of our solution set, we construct a channel assignment matrix, which remove non active links. This matrix consists of binary values $[0,1]$

$$
D_{c}=\left\{d_{m, n, c}\right\} \quad\{0,1\}
$$

Thus it can be said that, when $d_{m, n, c}=1$, then the link between transmitter $m$ and receiver $n$ will be part of a bunch of potential links for the channel c. if not, $\mathrm{d}_{\mathrm{m}, \mathrm{n}, \mathrm{c}}$

Will be nullified. In order to put a check on link conflicts, we impose the following condition on the spectral environment.

$$
\begin{aligned}
& a_{m, n, c}+a_{p, q, c} 1 \\
& \text { if } G_{c_{m, n, p, q}}=1 \\
& 1 \quad m, n, p, q \quad N
\end{aligned}
$$

We can determine the total network capacity of the spectral environment by taking the sum of product of channel assignment matrix and link capacity dratrix. Mathematically,

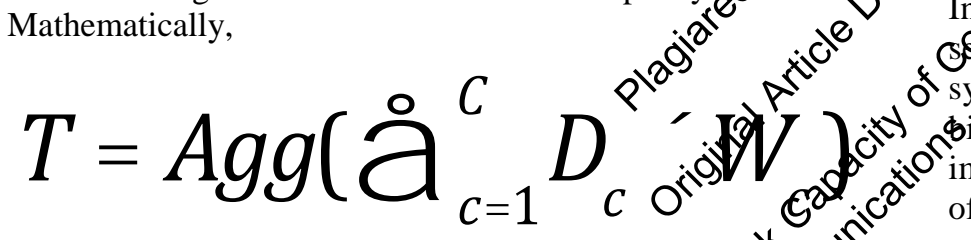

st

In the above equation, Agg is the operator that the addition of all entries of the matrix. $T$ jindicafes the overall network capacity of the spectral enjonent. It isd observed that $\mathrm{D}_{\mathrm{c}}$ and $\mathrm{W}_{\mathrm{c}}$ have uniform dipetion. The above equation can be made as an otptintzed equfeion which can be used as an objective functigro in pin on evolutionary algorithm such as gêtètionalgorithm. $x^{2} \lambda^{2}$ 4.3 Genetic Algorithm Gerstracetion:

In order to optimize the 5 extrum, we need sloysomplex interactive algorith horce we sort

algorithm. Each chromosome in genetic algorithm depicts an optimum solution from the total set of solutions.

Orderly arranging the rows of the assignment matrix Dc of multiple channels side by side to form a string having binary format builds chromosomes. For example, let us take three matrices namely P4, P5, P6 indicating the links on the channels 4,5,6:

$$
P_{4}=\left(\begin{array}{lll}
0 & 1 & 0 \\
0 & 0 & 1 \\
0 & 1 & 0
\end{array}\right)
$$

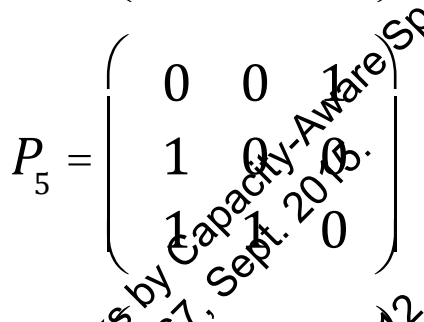

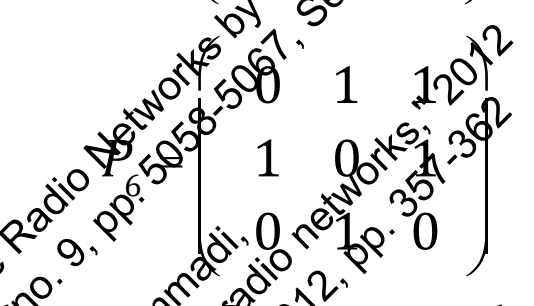

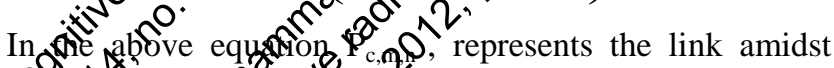
secendary useorming if it is unity. Hence, we sue maticall cgofnecoll the matrices into a string of binary format of length 27 bits. Since our motive is to

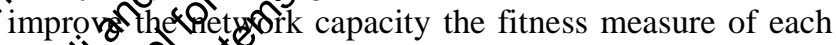
of do ditonsome implies the capacity of feasible metrotion of genetic algorithm, of chromosomes is sorted in accordance with their hesures. The chromosomes with improved fitness 10 anctobtained in the subsequent generations. The below \& gure shows crossover points on the edges between the links which indicate that they are from different channels. The main utility of these crossover points are the truncated co-channel conflicts shall not be carried to the subsequent generation.

Table 4.1: Crossover Points

\section{CROSSQVER POINTS}

$\mathrm{xx} \mathrm{d}$ $\mathrm{d}^{0}$

5ीANNEL 4 LINKS | CHANNEL 5 LINKS | CHANNEL 6 LINKS

\subsection{Codes and Standards}

Government authorities like Federal communication commission control the EM spectrum. Under the federal radio act of FCC, only the licensed users who are the authorized personnel can use the EM spectrum. The misuse of the EM Spectrum results in the production of Spectral efficiency of the wireless networks. To solve the spectrum under utilization problem, cognitive radio has been introduced. A cognitive radio should be cognizant of regulatory policies enforced by the licensing authorities. Cognitive radio is a self-functioning system hence it doesn't need any monitoring and can manage the wireless networks on its own. For reliable communication to happen, it is mandatory for QoS necessities to be met. Hence we sort it out for cognitive radio. In other words, 


\section{International Journal of Science and Research (IJSR) \\ ISSN (Online): 2319-7064}

Index Copernicus Value (2013): 6.14 | Impact Factor (2015): 6.391

QoS is described as calculable and filled with high quality features that are needed to accomplish the working of a function. Before the advent of cognitive radio, Software defined radio was being used for a wide range of operating frequencies. SDR is a radio terminal, which is capable of functioning in different bandwidths. To counter the drawback of an SDR i.e. QoS necessities are not met in a SDR hence it directed to the improvement of CR. The subsequent three meanings were additional to the SDR to make it a CR:

- Spectrum Management

- In line with a wide range of wireless networks and humans too thus delivering reserves to help humans in their activities.

\section{Schedule Tasks and Milestones}

Our Project journey was embarked by first being aware of the current work in Cognitive Radio and how it is being used to optimize the Spectral Environment. We first tried to understand the problem statement of our project. So that we can ponder over and arrive at an optimum solution.

Spectrum Optimization through dynamic spectrum access is our problem statement. Network Capacity has to be improved and the betterment of QoS parameters necessary to ensure reliable communication. We sorteft out for evolutionary algorithms to optimize the jective function (Total Network Capacity). We foug that bide encouraged algorithms were promising ideterming optimum solution for the given objective function:

Essential tasks of our project are to eliminate Qhite space from the spectral environment by opportunistallw accessing the spectrum, this is done by secondast yors who approach the band when it is unexpla of the primary users. The primary users and the sesondasey users should be topographically located so thet pow levels are within the bounds as discusstier. SIN of secondary users should have a mindumbalue of $\Delta^{5}$ Eger

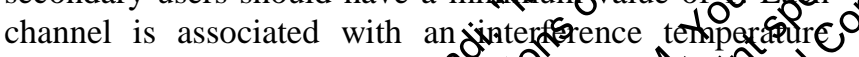
threshold, which should not of secondary users should rate the transmissionspot in accordance with the consfaintor

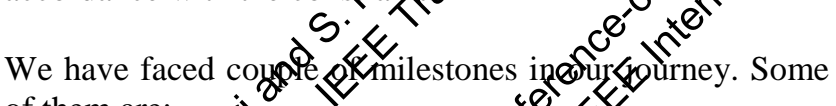
of them are:
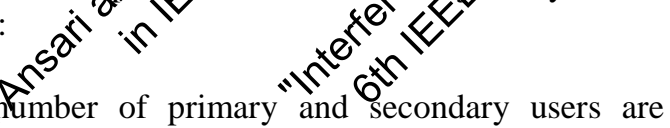

- As the number of primary "and secondary users are incrgased, the elapsed time in converging to an optimum sobntion will increase exponentially

$\$$ y increasing the number of active links, disrespecting their capacities heads to ruining of the overall network capacity.

- Genetic algorithm has an intrinsic trait of being random in nature. Hence due to the operations like mutation and crossover, by increasing the number of iterations does not necessarily assure us of an optimum solution.

- We also cannot ignore the time complexity in the functioning of the genetic algorithm.

\section{Project Demonstration}

We have built an environmental setup comprising of 4 primary users and 16 secondary users, which are spread across an area of 1024, square meters, as shown in Fig 6(a). As discussed earlier, we shall operate the cognition cycle stages to this spectral environment. With a supposition that secondary users operate through six operational channels. We have restricted interference on " each of the channels by imposing an interfereng temperature limit of $10 \mathrm{db}$ on each of the channels. Alş6 have a requirement of at least unity as the SINR.

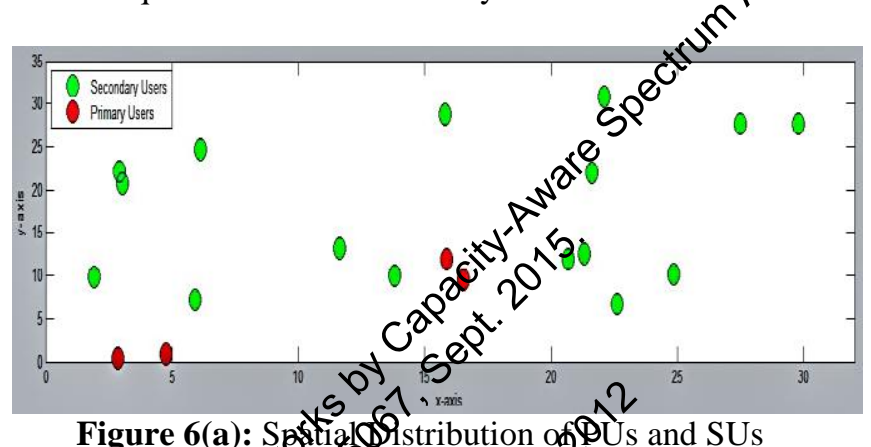

Figure 6(a): Sgetiano

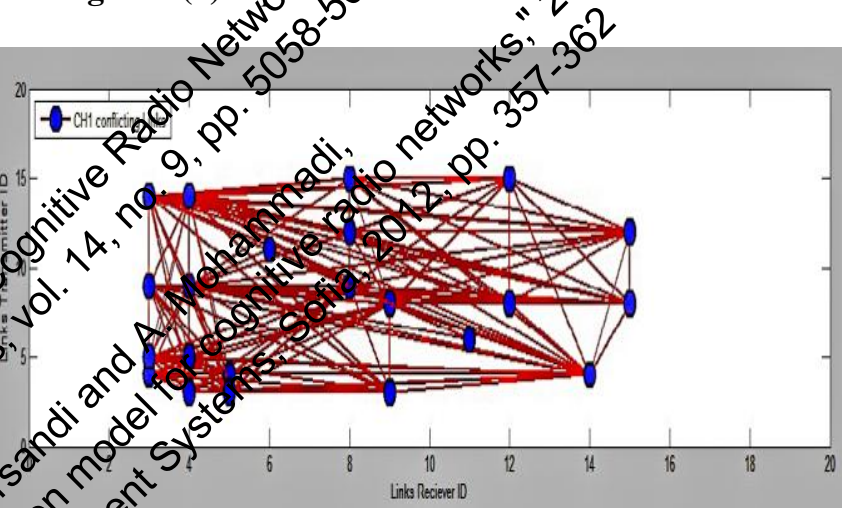

:

Eor trustworthy transmission, we initially spot the potential links and determine their network capacities and SINRs. We neglect the links with SINR less than 1 . We build an interference graph for each channel as shown in Fig 6(b), which represents the interference graph of channel 1. It is observed that genetic algorithm converges to an optimum solution as the number of iterations increases as shown in Fig 6(c).

We take another case where we have 25 secondary users and 5 primary users, spatially distributed across 2500 square meters. It has been observed that in this scenario that by increasing the number of active links among the secondary users, it need not certainly improve the network capacity by a significant factor as shown in fig6(d). The network capacity may increase only if we consider all the links as uniform, which is not practical. Intrinsic trait of genetic algorithm is highly arbitrary due to processes like crossover and mutation. 


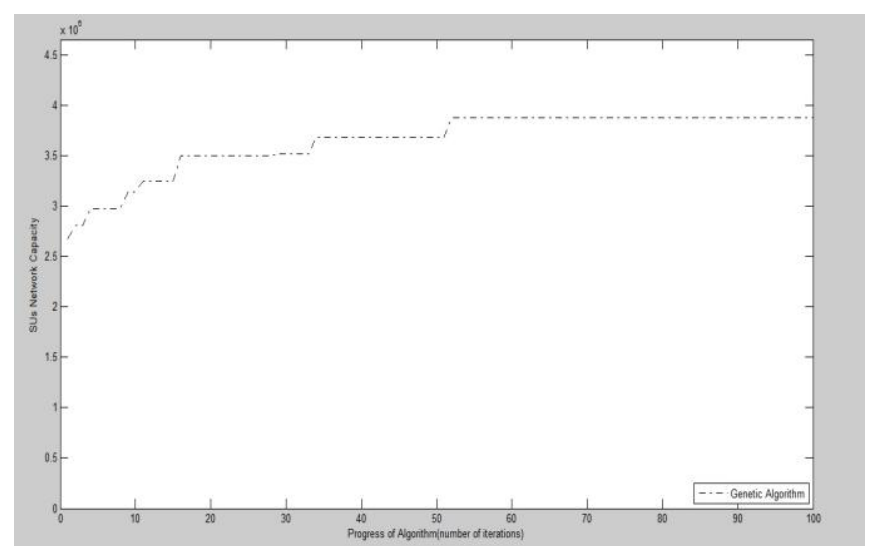

Figure 6(c): Total Network Capacity of the Secondary Users.(scenario-I)

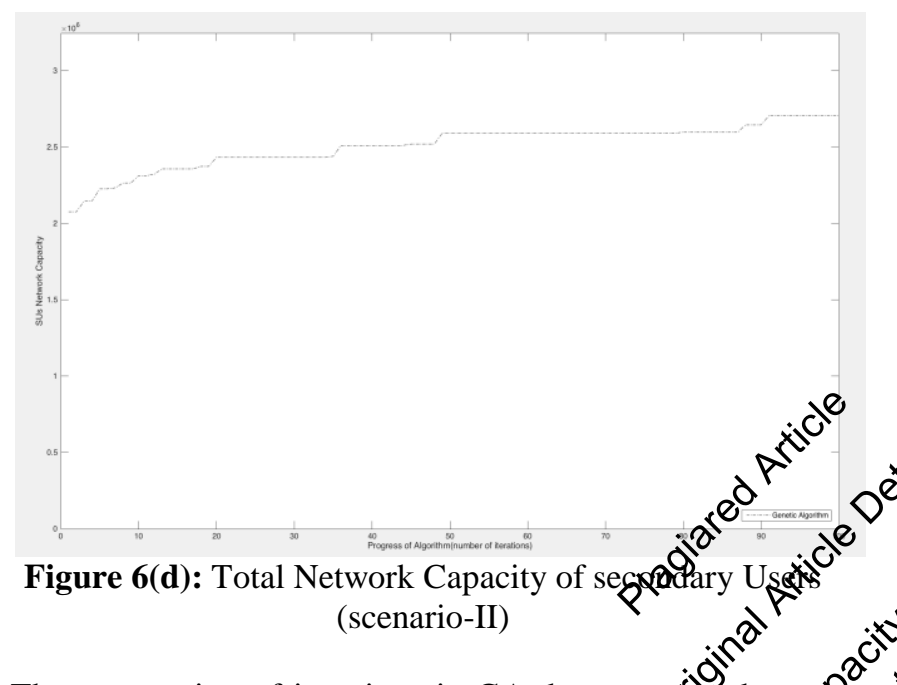

The progression of iterations in GA does nothandate optimum solution, which is evident from the pejiodiç? movement in the coming generations. In our pxoposet model, we have distributed the nodes in randea fastion due to which secondary users can control thecranconission power. Decreasing the distance betweerithegecondare users in populated areas, which in turnngutses thestoik SINR. Also, does this; there is morgatumber of linjes the

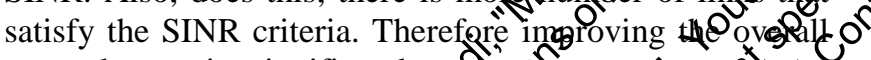
network capacity significantly

\section{Summary}

By providing opportunis access to yefole and available channels for the spendary userstectechnology of Cognitive Ras proves itself to hisly promising. And in that matter highly helps in making the usage of spectrum better. By optimization of variable number of paraneters, the enhancing of spectrum is met with and costlucted. In our project we have come up with little s f terference limited capacity known spectrum allowed ${ }^{\circ}$ design, to facilitate maximum capacity in Cognitive Radio.

The results shown in the simulation prove that it is able to achieve higher network capacity by inculcating the suggested model than the normal methods used for allocating the spectrum wisely. It has also come to our observation that, by maximizing the number of links that are active amongst the secondary users, it need not directly lead to the increase or maximization of the capacity of the network. It could be taken like that if we make an

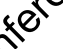

assumption that all the links are equal. But that form of an assumption would not be pragmatic to the approach of our scenario. We have also noticed that the scenarios in which the secondary users are equipped with the capability to control power, it is able to achieve higher capacity; given that the secondary users are distributed in a random manner as compared to the case in which they are laid out in a definite manner.

\section{References}

[1] I.F. Akyildiz, W. Lee, M. C. Vuran, and S. Mothanty, "Next generation/ dynamic spectrum accessognitive radio wireless networks: A survey," Cords sut. Netw., vol. 50, no. 13, pp. 2127-2159, Sep. 2epo.

[2] M. Yousefv and, S. Khorsandi, ande A. Mohammadi, "Interference- constraint spectrus allocation model for cognitive radio networks,, in Proc. 6th IEEE IS Conf., Sep. 6-8, 2012, pp. 302.

[3] Hill, R.R. "A Monte Car stuaty of genetic algorithm initial population geferate methods." Simulation Conference ProceęAngs, Phoenix, AZ: IEEE, 1999. 543-547.

[4] Mohammad tougeP and, Nirwan ansari, Fellow, IEEE, ant जै।

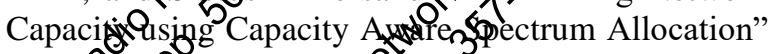
IEER 2 Trastion, on $n$ Wirgless Communications

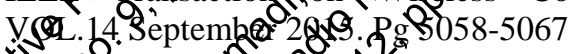
[5] o s. Wireldsín Nelw Pancouver, BC, Canada, 2007, pp. 2. [6] P. gruptoland ${ }^{9}$.'R. Kumar, "The capacity of wireless ditetys she Trans. Inf. Theory, vol. 46, no. 2, pp.
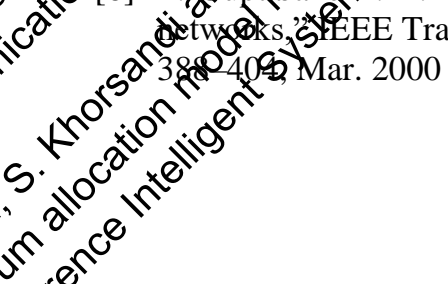